\title{
The hidden geometry of ocean flows
}

\author{
Carolina Mendoza and Ana M Mancho 1 \\ Instituto de Ciencias Matemáticas, CSIC-UAM-UC3M-UCM, Serrano 121, 28006 Madrid, Spain.
}

(Dated: November 5, 2018)

\begin{abstract}
We introduce a new global Lagrangian descriptor that is applied to flows with general time dependence (altimetric datasets). It succeeds in detecting simultaneously, with great accuracy, invariant manifolds, hyperbolic and non-hyperbolic flow regions.
\end{abstract}

PACS numbers: $\quad 02.30 . \mathrm{Hq}, 05.45 .-\mathrm{a}, 47.52 .+\mathrm{j}, 05.60 .-\mathrm{k}, 92.10 . \mathrm{Lq}$

Introduction.- - Finding order in the apparent disorder of ocean motion is still an open problem, as even flows that in their Eulerian description are smooth present messy float trajectories [2]. Typical ocean structures are eddies and currents. Major currents, such as the Gulf stream or the Kuroshio, impact the Earth's climate because of the heat they transfer. Eddies or rings are certain robust, long-lived structures that may travel hundreds to thousand of kilometres, and persist for periods lasting from months to years. Understanding transport across these large scale structures is a challenging task, but one increasingly amenable to treatment since now data are becoming available 2]. Lagrangian tools provide a characterization of fluid flows. Underlying their description is Poincaré's idea of seeking geometrical structures on the ocean surface (the phase portrait) that can be used to organize particles schematically by regions corresponding to qualitatively different types of trajectories. For stationary flows the fixed points are key for describing the solutions geometrically. Fixed points may be classified as hyperbolic or non-hyperbolic depending on their stability properties. Hyperbolic fixed points are responsible for particle dispersion and non-hyperbolic fixed points are related to particle confinement. The interplay between dispersion and confinement is an essential element of fluid transport processes. Stable and unstable manifolds of hyperbolic fixed points divide the phase portraits in regions with qualitatively different types of trajectories since they are barriers to transport. This letter describes a new Lagrangian descriptor that for flows with a general time dependence realizes Poincare's idea of dividing a phase portrait in different regions that correspond to trajectories with qualitatively different behaviours. Our new instrument is based on a function which has been introduced in 3 as a building block of a new definition of a Distinguished Trajectory (DT), which is a generalization of the concept of fixed point for aperiodically time dependent flows. Our function reflects, at the level of the phase portrait, relevant dynamical features of arbitrary time dependent dynamical systems. Some of these features have not previously been detectable, thus, when applied to altimetric ocean data sets, $M$ reveals the hidden geometry of the ocean flow. The technique locates simultaneously hyperbolic and non-hyperbolic flow regions. Since it reveals singular features along the stable and unstable manifolds of the Distinguished Hyperbolic Trajectories (DHTs), it is useful as well to detect these invariant curves, that near the DHTs coincide with its stable and unstable directions.

The function $M$.- The function we propose as a global Lagrangian descriptor (see [3]), considers the system:

$$
\dot{\mathbf{x}}=\mathbf{v}(\mathbf{x}, t), \mathbf{x} \in \mathbb{R}^{n}, t \in \mathbb{R}
$$

where $\mathbf{v}(\mathbf{x}, t)$ is $C^{r}(r \geq 1)$ in $\mathbf{x}$ and continuous in $t$. Let $\mathbf{x}(t)$ denote a trajectory and denote its components in $\mathbb{R}^{n}$ by $\left(x_{1}, x_{2}, \ldots, x_{n}\right)$. For all initial conditions $\mathbf{x}^{*}$ in an open set $\mathcal{B} \in \mathbb{R}^{n}$, at a given time $t^{*}$, we define the function $M\left(\mathbf{x}^{*}, t^{*}\right)_{\mathbf{v}, \tau}:(\mathcal{B}, t) \rightarrow \mathbb{R}$ for the system (1) as follows:

$$
M\left(\mathbf{x}^{*}, t^{*}\right)_{\mathbf{v}, \tau}=\int_{t^{*}-\tau}^{t^{*}+\tau}\left(\sum_{i=1}^{n}\left(d x_{i}(t) / d t\right)^{2}\right)^{1 / 2} d t
$$

In our observational oceanographic flow, particle advection occurs mainly in 2D (see 4 ), so $n=2$ in (1). $M$ is then the function that measures the Euclidean arc-length of the curve traced by a trajectory passing through $\mathbf{x}^{*}$ at time $t^{*}$ on the plane $\left(x_{1}, x_{2}\right)$. The trajectory is integrated from $t^{*}-\tau$ to $t^{*}+\tau$. The function $M$ depends on $\tau$ and also on the vector field $\mathbf{v}$. It is applicable to both time dependent and stationary flows. In the latter case it provides a time independent partition of the phase portrait. For instance, for the unforced, undamped Duffing equation a contour plot of $M$ depicts the familiar stable and unstable manifolds of the fixed point located at the origin. For time dependent flows the phase space partition provided by $M$ is time dependent.

The data set and the dynamical system. - The velocity data used in this work are geostrophic surface currents computed at CLS Int Corp (www.cls.fr) in the framework of the SURCOUF project [5]. The data span the whole Earth, at a resolution of $1 / 3$ degrees at Equator, but we focus our results over a region through which passes the Kuroshio current, in selected days of May and June 2003. Details on the data may be found in [6]. It is interpolated following methods described in [7, 8], that use bicubic interpolation in space and Lagrange polynomials in time. Our coordinate system $(\phi, \mu)$, is related to the longitude and latitude $(\phi, \lambda)$ by means of a transformation $\mu=\mu(\lambda)$ (see details in [6, 7]). These variables are convenient for they distribute the data on a uniform grid. The equations of motion for $(\phi, \mu)$ are,

$$
\frac{d \phi}{d t}=\frac{u(\phi, \mu, t)}{R \cos (\lambda(\mu))}, \quad \frac{d \mu}{d t}=\frac{v(\phi, \mu, t)}{R \cos (\lambda(\mu))}
$$



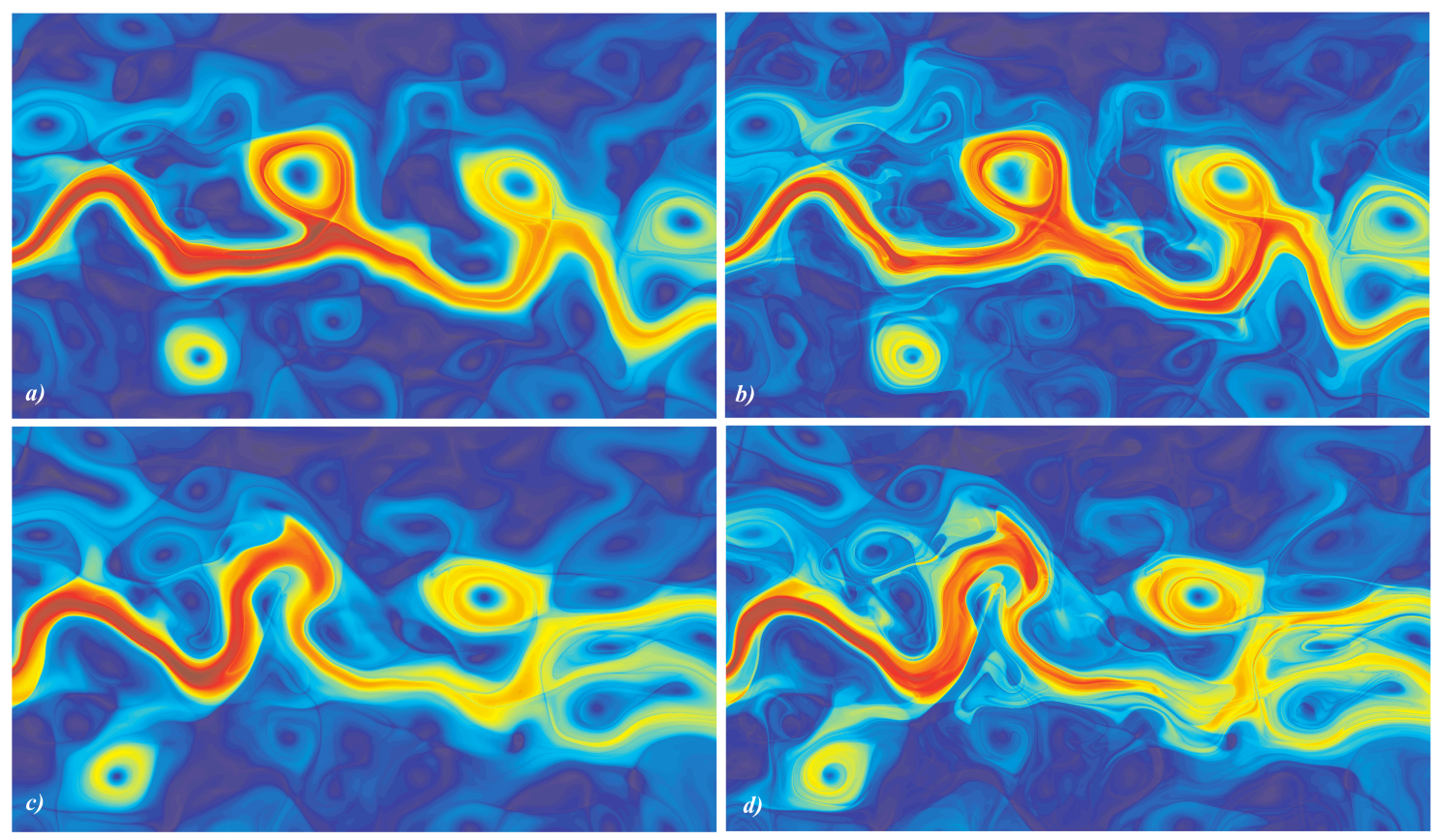

FIG. 1: Evaluation of the function $M$ over the Kuroshio current between longitudes $148^{\circ} \mathrm{E}-168^{\circ} \mathrm{E}$ and latitudes $30^{\circ} \mathrm{N}-41.5^{\circ} \mathrm{N}$; a) and b) on May 2, 2003; c) and d) on June 3, 2003. Panels a) and c) take $\tau=15$; Panels b) and d) take $\tau=30$.
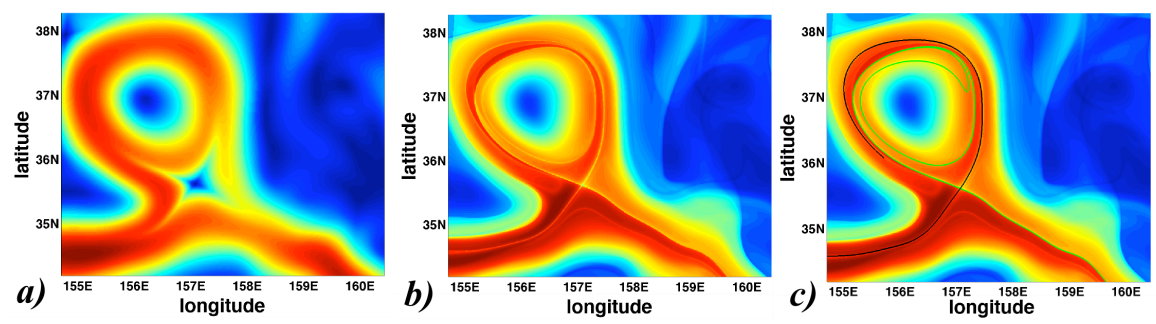

FIG. 2: The function $M$ on May 2, 2003. a) $\tau=2$; b) $\tau=15$; c) the same as b) with a piece of stable manifold (black) and a piece of unstable manifold (green) of the DHT overlapping.

where $u$ and $v$ represent the eastward and northward components of the altimetry surface velocity field respectively, and $R$ is the radius of the Earth. The factor, $1 / \cos (\lambda(\mu))$, in the $\mu$ equation is an artefact of the coordinate transformation. The function $M$ in Eq. 2 is computed over the dynamical system (3). Thus the length of the trajectory is measured on the $(\phi, \mu)$ plane. The system expressed in Eq. (3) is not exact, as it is subject to errors coming from the measured velocity fields, the sort of interpolation used, etc. However Eq. (3) is used for evaluating $M$, a function that contains Lagrangian information. In the literature [9], has been studied the robustness of the Lagrangian structures under errors induced in the vector field satisfying certain conditions. We have assessed the reliability of $M$ by computing it with several interpolation schemes.

Results.-We demonstrate that the function defined in Eq. (2) gives a global dynamic picture of oceanic flows since it detects simultaneously invariant manifolds, hyperbolic, and non-hyperbolic flow regions. It synthesizes information more efficiently than for instance spaghetti diagrams. These represent paths over time of messy trajectories but they do not communicate information about regions in which particle evolutions are qualitatively different, and one cannot get much intuition from them. Figure 1 displays the function $M$ for medium and large $\tau$ on selected days of May and June 2003 along the meandering Kuroshio current. Maximum values of $M$ are in red, while dark blue indicates minima. The dependence of $M$ on time is obvious for this highly aperiodic flow, since representations for different days have different structures. In the figure the organizing centres are visible at a glance. These key points are the minima of $M$, and as discussed in 3 they are related either to hyperbolic or non-hyperbolic distinguished trajectories. Singular features of $M$ forming lines are easily discerned, both in Figs. 1 and 2 Fig. 2p) shows their intersection at a hyperbolic minimum at longitude $\sim 157.1^{\circ}$ and latitude $\sim 35.63^{\circ}$. The time evolution of this point has been characterized in [6] as a DHT. Singular lines are identi- 

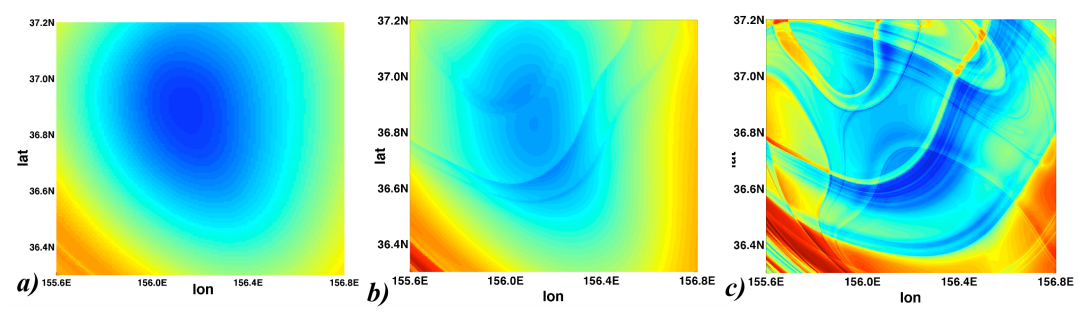

FIG. 3: The function $M$ evaluated over the inner core of an eddy on May 2, 2003. a) $\tau=15$; b) $\tau=30$; c) $\tau=72$.

fied as manifolds since they are advected by the flow and are asymptotically obtained from small segments aligned with the stable and unstable subspaces of the DHT. Fig. 2 c) shows the overlapping of $M$ with the stable and unstable manifolds computed with the technique used in [4. This confirms the coincidence of the lines with the manifolds. Why should stable and unstable manifolds be traced out by singular features of $M$ ? $M$ measures the lengths of curves traced by trajectories on the phase space, so it is expected it will change abruptly at the boundaries of regions comprising trajectories with qualitatively different evolutions, since this is exactly what the stable and unstable manifolds separate. Convergence of the structure of $M$ towards these singular lines requires a large enough $\tau$ value. For instance for $\tau=2$ the appearance of $M$ in Fig. 2 2 ) is rather simple, almost without structure and resembling that of Eulerian currents, while sharp lines in Fig. 2 b) and c) require the use of $\tau=15$. The structure of $M$ becomes more and more refined for larger $\tau$ values as confirmed by panels $1 \mathrm{p}$ ) and d), obtained for $\tau=30$. This is justified because $M$ reflects the history of initial conditions on open sets, and in highly chaotic systems this history is expected to be more complex for longer time intervals. The evaluation of $M$ in large oceanic areas as shown in Fig. 1 reveals recognizable phase portraits similar to those of the cat's eyes of the forced pendulum (in panel a) upper left), or the forced Duffing equation (see panel c) at the lower right). The ocean surface resembles a patchwork of interconnected dynamical systems from which the complexity of possible particle routes is visible.

In figures 1 and 2, apart from the minima of the function $M$ at the intersections of singular lines, related to the hyperbolic DT, there are apparent minima at the eddy centres. In the work by Madrid and Mancho [3] these have been related to non-hyperbolic DT (DET), which are eddy-like structures, of great interest to oceanographers. The Lagrangian description of eddies, such as that shown in Fig. 2 reveals the existence of an inner core, which is robust and rather impermeable to stirring and an outer ring, where the interchange with the media is understood in terms of lobe dynamics (see [10]). We analyze how the function $M$ reflects to what extent the inner core of Fig. 2 is impermeable to mixing. Figure 3 displays contour plots of $M$ on $t=$ May 2, 2003 for several $\tau$. In Fig. 3a) it is observed that for $\tau=15$ days the interior of the eddy has a minimum which is locally smooth. This means that in the range $(t-\tau, t+\tau)$ trajectories in this neighbourhood outline similar paths, and for this reason the function $M$ does not change sharply (i.e does not have singular features). Smoothness of $M$ implies that for these initial conditions it does not perceive nearby hyperbolic regions for $(t-\tau, t+\tau)$. Hyperbolic trajectories are the ones responsible for dispersion and it is just these trajectories that may induce sharp changes in $M$. In Figures 3b) and c) for larger $\tau$ values (i. e, $\tau=30$ and 72 respectively) the interior of the eddy becomes less and less smooth, for in the range $(t-\tau, t+\tau)$ trajectories placed at the interior core either were dispersed in the past or will disperse in the future. In fact, in Figure 3c), the interior of the core is completely foliated by singular features associated either to stable or unstable manifolds of nearby hyperbolic trajectories. So, the value at which $M$ starts losing smoothness, e.g. $2 \tau=60$, is a good indicator of the maximum time for confinement of particles in the inner core. The minimum of $M$ on the elliptic region does not converge with $\tau$, and this is the condition required for finding DT. Similarly to what is described in [3], DET have not been found in highly aperiodic flows. Figure 3p) displays in black line with a computed unstable manifold which overlaps on the contour plot of $M$. Again there is observed a coincidence of the singular features of $M$ with the manifold. However the foliated structure of $M$ is much richer than that provided by the manifold. The reason is that the manifold has been computed from the one DHT recognizable in Fig. 2b) and c), while $M$ displays all stable and unstable manifolds from all possible DHTs in the neighborhood of the eddy, without need for identifying DHTs a priori, as required by the manifold algorithm (see [4). Thus $M$ provides a complete partition of the phase portrait, while the direct computation of a manifold of a DHT does not.

The Lagrangian method using $M$ has several advantages over other methods based on finite time versions of Lyapunov exponents (LE) such as FTLE or FSLE [11, 12. LE techniques provide information on the linearised flow along trajectories and their focus is on hyperbolic regions. Ridges of FTLE and FSLE fields represent manifolds as reported for instance in [11, 12]. Figure 4 confirms this point. In it there is displayed the same eddy of Fig. 3 , but for $\tau=50$. The sharpest ridges of the FTLE field represented in Fig. 4b) and c) are 

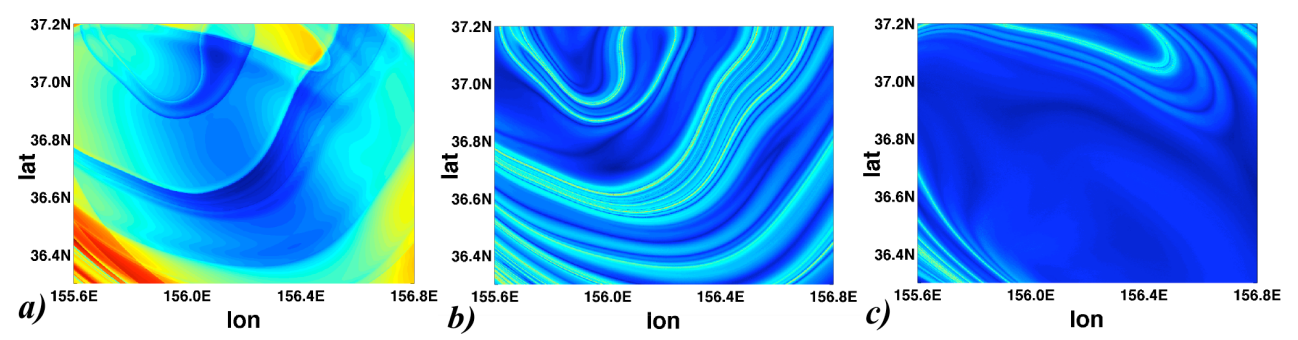

FIG. 4: Comparison of diverse Lagrangian techniques for $\tau=50$ at the same area of Fig. 3 ; a) The function $M$; b) the forward FTLE field; c) the backward FTLE field.

associated to the stable (forward FTLE) and unstable (backward FTLE) manifolds respectively, in close correspondence to the singular features of $M$ displayed in Fig. 4 a). An obvious difference between these two representations is that $M$ contains the information on both stable and unstable manifolds in the same picture, while FTLE splits it in two diagrams. Regarding other features provided by FTLE and $M$ there is not strict agreement. In the regions centered at longitude $\sim 156.1^{\circ}$ and latitude $\sim 36.8^{\circ}$ and at longitude $\sim 156.3^{\circ}$ and latitude $\sim 36.5^{\circ}$, the structure of $M$ is smooth and eddy-like, which as explained above, indicates that particles in that area do not disperse. The same areas in Fig. 4 b) display a striped pattern suggesting that particles disperse in this time interval. Numerical integrations of trajectories in these regions confirm that they stay close to each other in the interval $(t-\tau, t+\tau)$. Non-sharp striped structures in Fig. 4 b) raise then the questions of the kind of information they provide and if there is an upper bound on $\tau$ for this vector field, beyond which the validity of some of the structures provided by the FTLE cannot be confirmed. In 13 it is reported that for particular transient flows FTLE may develop 'ghost' structures, although a detailed discussion on this is beyond the scope of our letter. A further difference is that FTLE require certain assumptions on the vector field (see [1]), while by construction the function $M$ is defined for a general time dependent vector field and there is required only existence and uniqueness of solutions for the system (3).
Computationally the evaluation of the function $M$ is cheaper than that of LE. In geophysical flows, both $M$ and LE require the performance of a large number of integrations on a dynamical system such as (3), where the vector fields are interpolated over a finite space-time grid. Interpolations make computations expensive and saving these at each time step is a convenient feature. Evaluation of the function $M$ fits this criterion better than LE as each point in the phase space requires of just one integration forwards and backwards in time. LE techniques however require more interpolations of the velocity field at each time step, either because they evaluate a separation rate among several trajectories, or because they compute the linearised flow around each trajectory.

The Lagrangian descriptor $M$ locates special organizing trajectories called DT as reported in 3]. However although there are references suggesting the ability of LE to achieve this goal [14, there are no published studies where this is discussed in detail.

In conclusion, this work demonstrates the efficiency of a new Lagrangian descriptor $M$, for identifying the essential dynamical features of general time dependent flows. $M$ is a promising tool for the estimation of transport in realistic flows, as recent articles [6, 15] have confirmed. Pursuing further the discussion on how it contributes to transport diagnosis, is beyond the scope of this letter.

We thank D. Fox for useful comments, computer support from CESGA and grants: Oceantech, I-Math C30104, MTM2008-03754, MTM2008-03840-E, Simumat.
[1] Corresponding author. E-mail: a.m.mancho@icmat.es.

[2] A. S. Bowen and et al., Nature 459, 243 (2009).

[3] J. A. J. Madrid and A. M. Mancho, Chaos 19, 013111 (2009).

[4] A. M. Mancho, D. Small, and S. Wiggins, Nonlinear Proc Geoph 11, 17 (2004).

[5] G. Larnicol and et al., Proc. 15 yrs progr. in Radar altimetry ESA Symp., ESA, Venice (2006).

[6] C. Mendoza, A. M. Mancho, and M.-H. Rio, Nonlinear Proc Geoph 17, 103 (2010).

[7] A. M. Mancho and et al., J Phys Oceanogr 6, 1222 (2008).

[8] A. M. Mancho, D. Small, and S. Wiggins, Comp. \& Flu- ids 35, 416 (2005).

[9] G. Haller, Phys. Fluids 14, 1851 (2002).

[10] A. M. Mancho, D. Small, and S. Wiggins, Physics Reports 437, 55 (2006).

[11] G. Haller, Chaos 34, 99 (2000).

[12] F. d'Ovidio and et al., Geophys Res Lett 31, L17203 (2004).

[13] M. Branicki and S. Wiggins, Nonlinear Proc Geoph 17, 1 (2010).

[14] M. Branicki and R. Malek-Madani, Nonlinear Proc Geoph 17, 149 (2010).

[15] C. Mendoza and A. M. Mancho, arXiv:1006.3496. (2010). 